\title{
Method of Moments Simulation of Modulated Metasurface Antennas with a set of Orthogonal Entire-Domain Basis Functions
}

\author{
Modeste Bodehou, Student Member, IEEE, David González-Ovejero, Senior Member, IEEE, \\ Christophe Craeye, Senior Member, IEEE, and Isabelle Huynen, Senior Member, IEEE
}

\begin{abstract}
A family of orthogonal, and entire-domain basis functions (named Fourier-Bessel) is proposed for the analysis of circular modulated metasurface (MTS) antennas. In the structures at hand, the MTS is accounted for in the Electric Field Integral Equation (EFIE) as a sheet transition impedance boundary condition (IBC) on top of a grounded dielectric slab. The closed form Hankel transform of the Fourier-Bessel Basis Functions (FBBF) allows one to use a spectral domain formulation in the Method of Moments (MoM) solution of the EFIE. Moreover, these basis functions are fully orthogonal, which implies that they are able to represent the global evolution of the current distribution in a compact form. FBBFs also present a better filtering capability of their spectrum compared to other well known orthogonal families such as the Zernike functions. The obtained MoM matrix is sparse and compact, it is thus very well conditioned and can be efficiently computed and inverted. Numerical results based on the proposed decomposition are presented and compared with those based on the use of Gaussian Ring Basis Functions (GRBF) and with full-wave analysis of MTS antennas implemented with small printed elements. A very good agreement is observed.
\end{abstract}

Index Terms-Method of Moments, basis functions, spectral domain methods, metasurfaces (MTSs), leaky-wave (LW) antennas, impedance boundary condition (IBC).

\section{INTRODUCTION}

I $\mathrm{N}$ recent years, a lot of interest has been attracted by artificial surfaces able to provide electromagnetic properties that one cannot find in nature [1], [2]. Such structures, commonly referred to as metasurfaces (MTSs), can be employed in a wide range of frequencies, from microwaves [3] to optics [4]. Although MTSs may also be used to tailor the transmission of space waves [4], [5], they have found a vast number of applications in the control of surface-waves (SWs) wavefronts [6], [7] and the design of aperture antennas [8]-[14]. At microwave frequencies, metasurfaces may be implemented as dense textures of sub-wavelength elements (typically $\lambda / 10$, with $\lambda$ being the free-space wavelength) printed on a grounded dielectric slab [10]. The resulting number of printed patches is generally around $10^{4}$ for devices with a $15 \lambda$ diameter. Indeed, MTS antennas [11] generally consist of circular apertures,

M. Bodehou, C. Craeye and I. Huynen are with the Universite catholique de Louvain, in the ICTEAM institute, Place du Levant 3, 1348 Louvain-la-Neuve, Belgium (e-mails: modeste.bodehou@uclouvain.be, christophe.craeye@uclouvain.be, isabelle.huynen@uclouvain.be).

D. González-Ovejero is with the Institut d'Electronique et de Télécommunications de Rennes - IETR, UMR CNRS 6164, Université de Rennes 1, 35042 Rennes, France (david.gonzalez-ovejero@univ-rennes1.fr). where a SW launcher [15] is used to excite the structure. This paper will focus on circular apertures, bearing in mind that a proper stretching of the proposed basis functions may enable the treatment of elliptical ones [12], [16], [17].

The numerical analysis of MTS devices is very challenging, due to the large number of electrically small printed elements. In fact, this feature leads to a very dense mesh (the example in [11, Sec. III.D] required about $10^{6}$ basis functions) and, hence, a prohibitive memory and simulation time for the resulting system of equations. Besides, the presence of fine mesh details can yield an ill-conditioned system of equations. Therefore, one has to combine iterative solvers with efficient preconditionners [18], use analytical [19] or characteristics basis functions [20], [21] to reduce the number of unknowns, or apply a combination of both [22]. However, analytical and characteristic basis functions may depend on the shape and the orientation of the patches and the aforementioned fast methods also become inefficient for apertures with diameters larger than $20 \lambda$.

Given the electrically small size of the patches, the fields in the cell allotted to each patch can be averaged, and the actual MTS structure can thus be represented by an equivalent impedance boundary condition (IBC) [1], [2]. The conditions under which the IBC model is applicable have been discussed in [23]. The synthesis problem then consists of finding the appropriate IBC which will perturb the original SW in order to generate the desired radiation or wave-guiding effect. In the case of antenna engineering, the interest lies in transforming the SW into a LW. Several methods have been proposed in the literature, ranging from holographic techniques [13] to the recent theory of Flat Optics (FO) [24], [14]. This paper does not address the design problem. However, a direct simulation of radiation by the modulated IBC is sufficient to provide an excellent idea about the performance of a given design [11], [25] and by considering the IBC problem, one can optimize a few parameters of the modulation before implementing it. Once the design has been completed, the MTS can be implemented by gradually changing the shape, dimensions and orientation of the sub-wavelength patches, under a local periodicity assumption [10]-[12].

MTS structures can be represented as IBCs in two different ways. In both cases, the obtained boundary condition is valid for a given incidence. The first possibility consists of describing the whole metasurface (MTS) as a unique entity, assuming its impenetrability. One can then represent the MTS 
in the form of an opaque or impenetrable IBC [26]-[27] which relates the average tangential electric field to the average tangential magnetic field at the upper interface (see Fig. 1(a) from [25]). The second possibility is to treat separately the

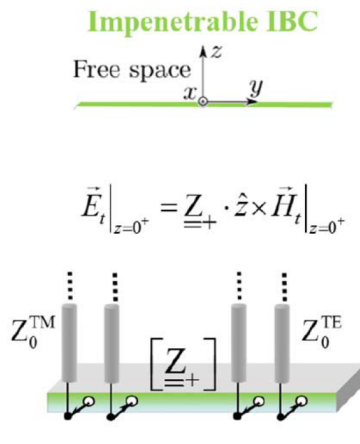

(a)

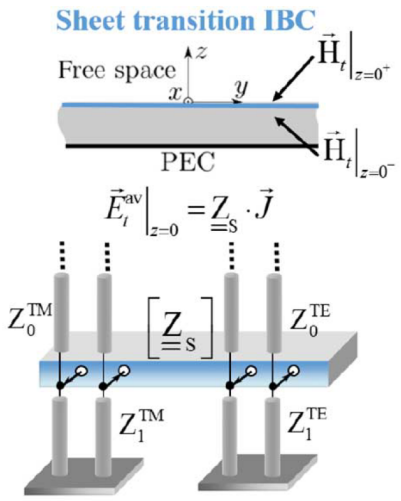

(b)
Fig. 1: From [25], equivalent transmission line circuits for the (a) impenetrable (opaque) IBC and (b) sheet transition IBC for grounded dielectric slab MTS.

cladding and the substrate [2], [26], [29]. The resulting sheet transition IBC now relates the average tangential electric field on the MTS sheet to the difference of the tangential magnetic fields on either side of the MTS cladding, which stands for the current flowing on the sheet (see Fig. 1(b) from [25]). The sheet transition IBC can be obtained from the opaque one, after removing the contribution of the substrate, as described in [30]. The stability issue of both representations has been discussed in [31]. Although both approaches present instabilities, the second formulation is more stable and accurate for practical values of the IBC. Moreover, in practical optimization problems, the substrate does not change and the optimization parameters are only related to the sheet impedance and to the excitation. For the aforementioned reasons, the sheet transition IBC formulation is selected in this paper.

The IBCs generally consist of smooth locally periodic modulations, with period of the order of the free-space wavelength. It is thus reasonable to expect that the currents flowing on the MTS sheet be also smooth and with variation at the wavelength scale. It should therefore be possible (by using entire-domain basis functions) to represent the current distribution with a number of basis functions drastically smaller than the one needed to mesh the patches with sub-entire domain basis functions. Such full-domain basis functions provide a good representation of the global field propagation along the surface, while local effects such as interaction with the feeding structure may be less accurate. The authors of [25] propose the use of Gaussian Ring Basis Functions (GRBF). These basis functions lead to a drastic reduction of the Method of Moments matrix size and a very short computation time. This family of functions tends to provide a good localization of rapid changes in surface currents, owing to the Gaussian decay of the functions along the radial coordinate around a given radius. In opposition, the family of functions proposed here has a relatively homogeneous level over the whole radial domain, which allows a good distinction between different "modes" of propagation over the whole structure. The goal pursued here is to represent more compactly the global evolution of the current distribution by using a set of fully orthogonal and entire-domain basis functions.

Two families of functions are well known, due to their orthogonality on a disk. The first one is the Zernike family of functions [32], [33], which present a polynomial radial dependence. The second one is the Fourier-Bessel family [34]. Both families possess a closed form spectrum, but the Zernike spectrum is much wider. This leads to a non-negligible asymptotic component (in the integrals describing the substrate matrix) that needs to be carefully taken into account by properly choosing the testing operation. Moreover, Zernike functions are less suited than the Bessel family to describe typical current distributions on MTS owing to their non-zero admissible edge currents. For the aforementioned reasons, Fourier-Bessel functions are more convenient to describe the current distribution on MTS antennas.

The primary goal of this paper is to demonstrate that an appropriate choice of full-domain basis functions can lead to more compact discretizations than previously used representations [25]. The second objective consists of providing an efficient formulation for the reaction integrals between the proposed basis functions for the electric-field integral equations (EFIE) in the presence of layered media. This paper may be viewed as an extension of a previous communication [35], in which the primary goal had not been addressed; it will also provide supplementary results regarding the efficient calculation of the reaction integrals.

The remainder of the manuscript is structured as follows. Section II presents the family of Fourier-Bessel Basis Functions as well as their spectral behavior. Section III describes the Method of Moments formulation. Section IV illustrates the numerical simulation of metasurface antennas, comparing them with Gaussian Ring Basis Functions (GRBF), already validated in [25] and with full-wave analysis of MTS antennas implemented with small printed elements. Finally, conclusions are drawn in section $\mathrm{V}$.

\section{Fourier-BESSEL BASIS FunCtions (FBBF)}

The circular MTS region is placed at the air dielectric interface of an infinite grounded dielectric slab, which corresponds to the $\mathrm{z}=0$ plane of a Cartesian coordinate system $(x, y, z)$ with unit vectors $\hat{a}_{x}, \hat{a}_{y}, \hat{a}_{z}$ (see Fig. 1(b)). The observation point on this surface will be denoted by $\vec{\rho}$. The FBBFs are described in cylindrical coordinates $(\rho, \phi)$, with unit vectors $\left(\hat{a}_{\rho}, \hat{a}_{\phi}\right)$, and accordingly, the observation vector is $\vec{\rho}=\rho \cos (\phi) \hat{a}_{x}+\rho \sin (\phi) \hat{a}_{y}$.

The Bessel functions are defined on a unit disk $\left(0 \leq \rho_{0} \leq 1\right)$ as follows [34]:

$$
F_{m, n}\left(\rho_{0}\right)=J_{n}\left(\lambda_{n}^{m} \rho_{0}\right)
$$

where $\lambda_{n}^{m}$ is the $m$-th positive zero of $J_{n}(x)$ (the Bessel function of the first kind and order $n$ ). This class of functions are orthogonal for a fixed value of $n$ (see Appendix A). In 
addition, this family of Bessel functions admits a closed form Hankel transform:

$$
\tilde{F}_{m, n}\left(k_{\rho}\right)=-\frac{\lambda_{n}^{m} J_{n-1}\left(\lambda_{n}^{m}\right) J_{n}\left(k_{\rho}\right)}{\left(\lambda_{n}^{m}\right)^{2}-k_{\rho}^{2}}
$$

Fig. 2(a) and Fig. 2(b) illustrate the behavior of the Bessel functions in (1) for different values of $m$ and $n$, respectively. The parameter $m$ controls the number of oscillations. The

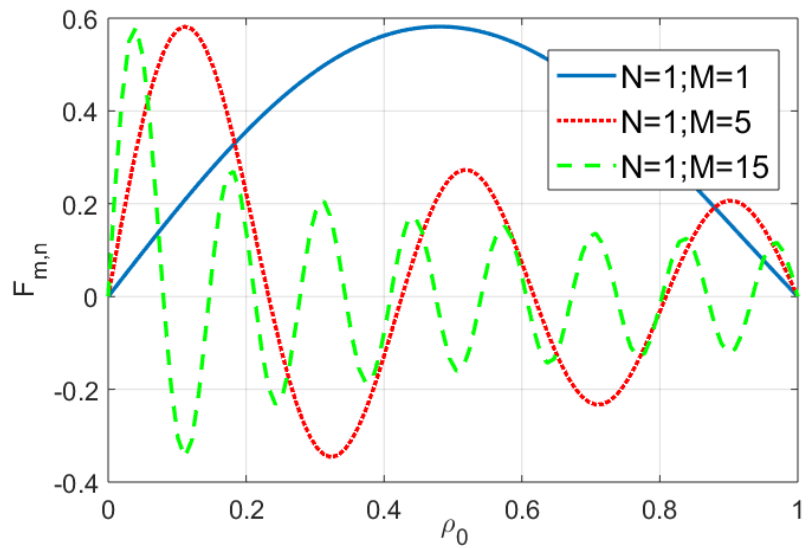

(a)

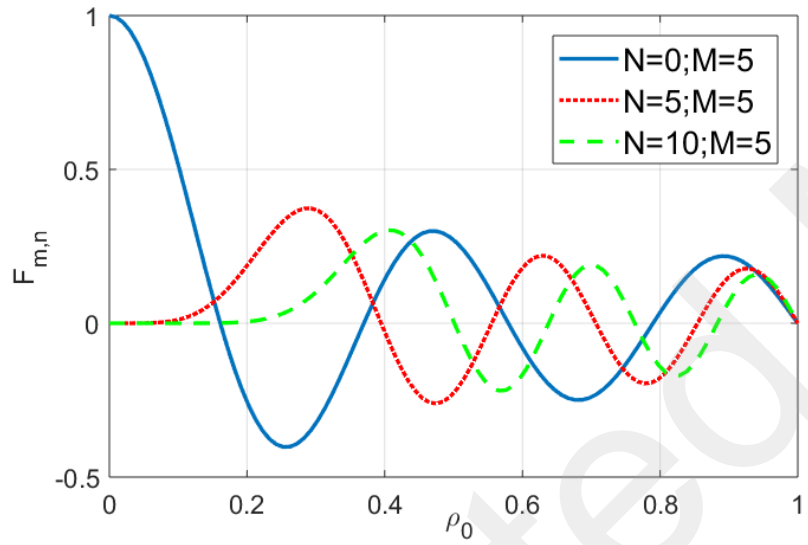

(b)

Fig. 2: Radial part of the FBBFs for (a) a fixed $n=1$ index and $m=1,5$ and 15 , (b) a fixed $m=5$ index and $n=0,5$ and 10 .

number of maxima and minima with positions different from $\rho_{0}=0$ and $\rho_{0}=1$ is equal to $m$, for any value of $n$. On the other hand, the parameter $n$ controls essentially the behavior near to the origin. Fig. 3(a) and Fig. 3(b), show the spectra which correspond to the Bessel functions plotted in Fig. 2(a) and Fig. 2(b), respectively. One can see that the spectrum oscillates until the peak at $k_{\rho}=\lambda_{n}^{m}$. From this value, it decays very fast. This results in an easy rule of thumb for the integration domain. It is important to note that the spectrum is finite at $\lambda_{n}^{m}$ since, the numerator is also equal to 0 .

The azimuthal dependence is introduced after multiplying $F_{m, n}\left(\rho_{0}\right)$ by $e^{-j n \phi}$, where $\phi$ is the azimuthal coordinate. The Fourier-Bessel Basis Functions (FBBF) are then defined on a disk of radius $a$ as:

$$
R_{m, n}\left(\frac{\rho}{a}, \phi\right)=F_{m, n}\left(\frac{\rho}{a}\right) e^{-j n \phi}
$$

This azimuthal behavior generalizes the orthogonality, even for different values of $n$. The obtained FBBF family is then fully

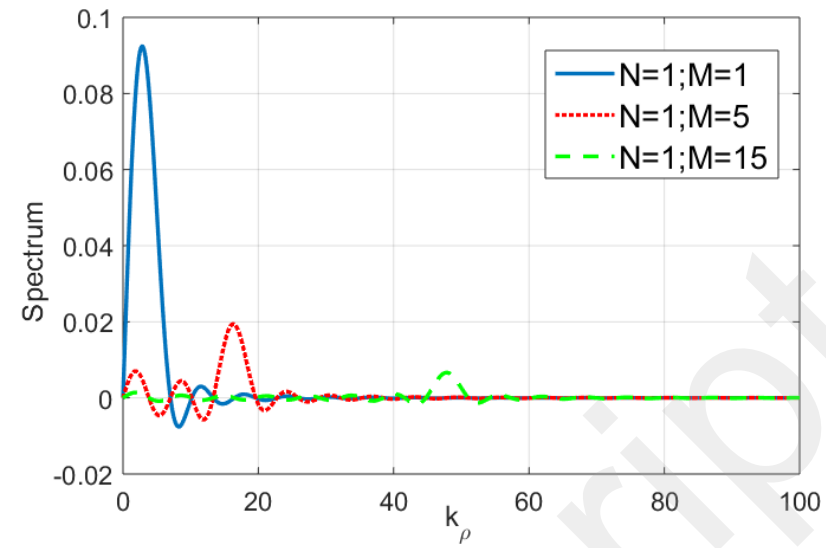

(a)

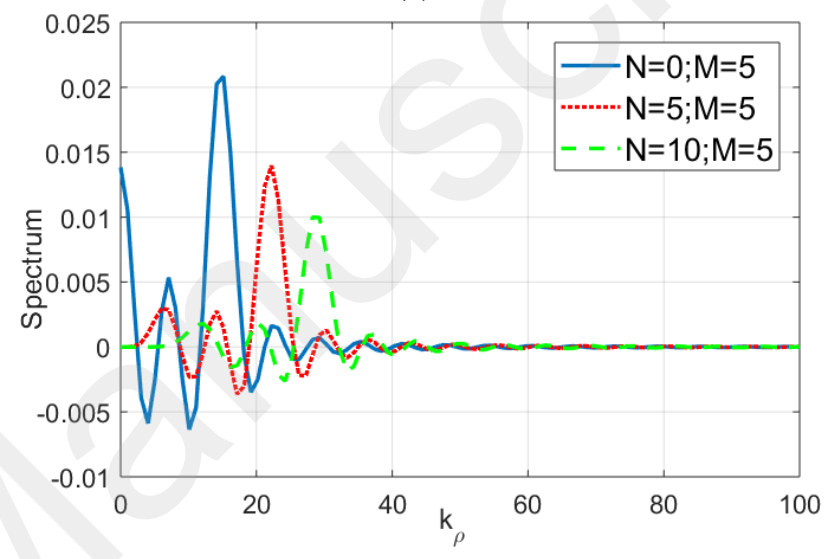

(b)

Fig. 3: Spectral domain counterpart of the radial part of the FBBFs shown in (a) Fig. 2(a) for a fixed $n$ index, (b) Fig. 2(b) for a fixed $m$ index.

orthogonal (see Appendix A) and their closed form Hankel transform is:

$$
\tilde{R}_{m, n}\left(k_{\rho}, \alpha\right)=-2 \pi j^{n} e^{-j n \alpha}\left[\frac{a^{2} \lambda_{n}^{m} J_{n-1}\left(\lambda_{n}^{m}\right) J_{n}\left(k_{\rho} a\right)}{\left(\lambda_{n}^{m}\right)^{2}-\left(k_{\rho} a\right)^{2}}\right]
$$

where $k_{\rho}$ and $\alpha$ are the spectral variables in cylindrical coordinates.

As observed, the FBBFs are directly defined in all the circular domain and vanish at the rim of the disc. That is, they do not admit a normal component at the border. This property ensures the absence of line charges at the border in the Integral Equation formulation.

The current distribution can be decomposed in two ways. First, by using its Cartesian $x$ and $y$ orthogonal components as follows:

$$
\vec{J}(\vec{\rho})=\sum_{n=-N}^{N} \sum_{m=1}^{M} i_{m n}^{x} R_{m, n}^{x}\left(\frac{\rho}{a}, \phi\right) \hat{a}_{x}+i_{m n}^{y} R_{m, n}^{y}\left(\frac{\rho}{a}, \phi\right) \hat{a}_{y}
$$

where $\hat{a}_{x}$ and $\hat{a}_{y}$ are the unit vectors along the $x$ and $y$ directions, respectively.

The second possibility consists in using the $\left(\hat{a}_{\rho}, \hat{a}_{\phi}\right)$ decomposition, which is useful to reduce the number of unknowns for isotropic MTS. This decomposition cannot be efficiently and directly used in a MoM formulation, as the resulting 
dyadic Green's function does not exclusively depend on the vector between the source and observation positions, but on both positions. However, owing to the orthogonality of the basis functions, the second formulation can be extracted from the first one, by decomposing the $\left(\hat{a}_{x}, \hat{a}_{y}\right)$ basis and testing functions into $\left(\hat{a}_{\rho}, \hat{a}_{\phi}\right)$ basis and testing functions, as explained in section III-D.

\section{Method of Moments Formulation}

Let us start from the definition of the sheet transition IBC:

$$
\vec{E}_{t}=\underline{\underline{Z}}_{S} \vec{J}
$$

where $\vec{E}_{t}$ is the tangential electric field on the MTS plane, and it stands for the sum of the E-field radiated by the equivalent currents $\vec{J}$ and that from the excitation. Equation (6) then leads to the following Electric Field Integral Equation:

$$
\hat{n} \times\left[\iint_{S^{\prime}} \underline{\underline{G}}^{E J}\left(\vec{\rho}, \vec{\rho}^{\prime}\right) \vec{J}\left(\vec{\rho}^{\prime}\right) d S^{\prime}-\underline{\underline{Z}}_{S}(\vec{\rho}) \vec{J}(\vec{\rho})\right]=-\hat{n} \times \vec{E}_{i}
$$

where $\hat{n}$ is the normal to the surface, $\vec{\rho}^{\prime}=x^{\prime} \hat{a}_{x}+y^{\prime} \hat{a}_{y}$ and $\vec{\rho}=x \hat{a}_{x}+y \hat{a}_{y}$ are the source and observation points, respectively. $\underline{G}^{E J}$ is the dyadic Green's function of the grounded dielectric slab, $\underline{\underline{Z}}_{S}$ is the sheet transition IBC tensor and $\vec{E}_{i}$ is the excitation electric field. The fields are tested with the complex conjugate of the basis functions. Nevertheless, testing with the basis functions themselves also provides good results. After introducing the current expansion (5) in (7) and testing the fields, (7) leads to a system of equations that can be written in a compact form as follows:

$$
\left[\begin{array}{ll}
{\left[Z^{x x}\right]} & {\left[Z^{x y}\right]} \\
{\left[Z^{y x}\right]} & {\left[Z^{y y}\right]}
\end{array}\right]\left[\begin{array}{l}
{\left[i^{x}\right]} \\
{\left[i^{y}\right]}
\end{array}\right]=\left[\begin{array}{l}
{\left[v^{x}\right]} \\
{\left[v^{y}\right]}
\end{array}\right]
$$

Identifying each testing function with the couple of parameters $(m, n)$ and each basis function with the couple parameters $\left(m^{\prime}, n^{\prime}\right)$, each element of the submatrix $\left[Z^{x x}\right]$ is defined as:

$$
Z^{x x}\left(m, n ; m^{\prime}, n^{\prime}\right)=Z_{G}^{x x}\left(m, n ; m^{\prime}, n^{\prime}\right)-Z_{I B C}^{x x}\left(m, n ; m^{\prime}, n^{\prime}\right)
$$

with:

$$
\begin{array}{r}
Z_{G}^{x x}\left(m, n ; m^{\prime}, n^{\prime}\right)=\int_{0}^{2 \pi} \int_{0}^{a} R_{m, n}^{x, *}\left(\frac{\rho}{a}, \phi\right) \\
\int_{0}^{2 \pi} \int_{0}^{a} G_{x x}^{E J}\left(\left|\vec{\rho}-\vec{\rho}^{\prime}\right|\right) R_{m^{\prime}, n^{\prime}}^{x}\left(\frac{\rho^{\prime}}{a}, \phi\right) \rho^{\prime} d \rho^{\prime} d \phi^{\prime} \rho d \rho d \phi
\end{array}
$$

and the sheet IBC contribution:

$$
\begin{array}{r}
Z_{I B C}^{x x}\left(m, n ; m^{\prime}, n^{\prime}\right)=\int_{0}^{2 \pi} \int_{0}^{a} R_{m, n}^{x, *}\left(\frac{\rho}{a}, \phi\right) Z_{S}^{x x}(\rho, \phi) \\
R_{m^{\prime}, n^{\prime}}^{x}\left(\frac{\rho}{a}, \phi\right) \rho d \rho d \phi
\end{array}
$$

The excitation vector $\left[v_{x}\right]$ is given by:

$$
v^{x}(m, n)=-\int_{0}^{2 \pi} \int_{0}^{a} R_{m, n}^{x, *}\left(\frac{\rho}{a}, \phi\right) E_{i}^{x}(\rho, \phi) \rho d \rho d \phi
$$

Finally, as the substrate Green's function is known in closed form in spectral domain, and the FBBFs admit a closed form Fourier transform, it is more convenient to evaluate the substrate and the excitation contribution directly in the spectral domain. Expressions (10) and (12) can be rewritten respectively as:

$$
\begin{aligned}
& Z_{G}^{x x}\left(m, n ; m^{\prime}, n^{\prime}\right)=\frac{1}{4 \pi^{2}} \int_{0}^{2 \pi} \int_{0}^{\infty} \tilde{R}_{m, n}^{x, *}\left(k_{\rho}, \alpha\right) \tilde{G}_{x x}^{E J}\left(k_{\rho}, \alpha\right) \\
& \tilde{R}_{m^{\prime}, n^{\prime}}^{x}\left(k_{\rho}, \alpha\right) k_{\rho} d k_{\rho} d \alpha
\end{aligned}
$$

$$
\begin{array}{r}
v_{x}(m, n)=-\frac{1}{4 \pi^{2}} \int_{0}^{2 \pi} \int_{0}^{\infty} \tilde{R}_{m, n}^{x, *}\left(k_{\rho}, \alpha\right) \tilde{E}_{i}^{x}\left(k_{\rho}, \alpha\right) \\
k_{\rho} d k_{\rho} d \alpha
\end{array}
$$

where $\tilde{G}_{x x}^{E J}\left(k_{\rho}, \alpha\right)$ is the $x x$ component of the Green's function spectral dyad and $\tilde{E}_{i}^{x}\left(k_{\rho}, \alpha\right)$ is the $\hat{x}$-oriented component of the excitation spectrum.

The elements in the others submatrices $\left[Z^{x y}\right],\left[Z^{y x}\right]$ and $\left[Z^{y y}\right]$ in (8) and that of $\left[v_{y}\right]$ are given by definitions analogous to (9), (11), (13) and (14).

\section{A. Grounded slab contribution}

The substrate interaction matrix is directly formulated in the spectral domain. The dyadic spectral Green's function of the grounded slab can be written as follows [36]-[38]:

$$
\left[\begin{array}{ll}
\tilde{G}_{x x}^{E J} & \tilde{G}_{x y}^{E J} \\
\tilde{G}_{y x}^{E J} & \tilde{G}_{y y}^{E J}
\end{array}\right]=\tilde{G}_{A}\left(k_{\rho}\right)\left[\begin{array}{cc}
1 & 0 \\
0 & 1
\end{array}\right]+\tilde{G}_{V}\left(k_{\rho}\right)\left[\begin{array}{cc}
k_{x}^{2} & k_{x} k_{y} \\
k_{x} k_{y} & k_{y}^{2}
\end{array}\right]
$$

where $\tilde{G}_{A}$ and $\tilde{G}_{V}$ are, respectively, the scalar potentials with respect to currents and charges. After substituting (15) into (13) and integrating along $\alpha$, the substrate matrix elements are given by:

$$
Z_{G}^{x x}\left(m, n ; m^{\prime}, n^{\prime}\right)=(-j)^{n} j^{n^{\prime}} a^{4} \int_{0}^{\infty} \tilde{F}_{m, n}\left(k_{\rho} a\right) \tilde{F}_{m^{\prime}, n^{\prime}}\left(k_{\rho} a\right)
$$$$
\left[2 \pi \tilde{G}_{A}\left(k_{\rho}\right) \delta_{n, n^{\prime}}+\tilde{G}_{V}\left(k_{\rho}\right) \epsilon_{n, n^{\prime}}^{c c}\right] k_{\rho} d k_{\rho}
$$

$$
\begin{gathered}
Z_{G}^{x y}\left(m, n ; m^{\prime}, n^{\prime}\right)=Z_{G}^{y x}\left(m, n ; m^{\prime}, n^{\prime}\right)=(-j)^{n} j^{n^{\prime}} a^{4} \\
\int_{0}^{\infty} \tilde{F}_{m, n}\left(k_{\rho} a\right) \tilde{F}_{m^{\prime}, n^{\prime}}\left(k_{\rho} a\right)\left[\tilde{G}_{V}\left(k_{\rho}\right) \epsilon_{n, n^{\prime}}^{s, c}\right] k_{\rho} d k_{\rho} \\
Z_{G}^{y y}\left(m, n ; m^{\prime}, n^{\prime}\right)=(-j)^{n} j^{n^{\prime}} a^{4} \int_{0}^{\infty} \tilde{F}_{m, n}\left(k_{\rho} a\right) \tilde{F}_{m^{\prime}, n^{\prime}}\left(k_{\rho} a\right) \\
{\left[2 \pi \tilde{G}_{A}\left(k_{\rho}\right) \delta_{n, n^{\prime}}+\tilde{G}_{V}\left(k_{\rho}\right) \epsilon_{n, n^{\prime}}^{s s}\right] k_{\rho} d k_{\rho}}
\end{gathered}
$$

with $\epsilon_{n, n^{\prime}}^{c c}=(\pi / 2)\left(2 \delta_{n, n^{\prime}}+\delta_{n, n^{\prime}+2}+\delta_{n, n^{\prime}-2}\right), \epsilon_{n, n^{\prime}}^{s c}=$ $(\pi / 2 j)\left(\delta_{n, n^{\prime}-2}-\delta_{n, n^{\prime}+2}\right)$ and $\epsilon_{n, n^{\prime}}^{s s}=(\pi / 2)\left(2 \delta_{n, n^{\prime}}-\right.$ $\left.\delta_{n, n^{\prime}+2}-\delta_{n, n^{\prime}-2}\right)$. The function $\delta_{i, j}$ is the Kronecker delta. These integrals can be efficiently computed using a parabolic contour deformation [39]. In practice, an integration range from 0 to $2 \lambda_{N}^{M} / a$ is enough to obtain accurate results. For examples discussed in this paper, $2 \lambda_{N}^{M} / a \approx 10 k_{0}$, with $k_{0}=2 \pi / \lambda$, where $\lambda$ is the free-space wavelength. This means that each integration can be carried out with about 500 sampling points by using a higher-order quadrature, such as 
the Gauss-Legendre one. The obtained substrate matrix $Z_{G}$ is relatively sparse since it has to be calculated for only few elements. This matrix structure has already been discussed in [25]. The substrate matrix can then be computed extremely fast (less than 1 minute on a laptop computer).

\section{B. Sheet impedance contribution}

The analysis is limited here to the $Z_{I B C}^{x x}$ component since the obtained expression is valid for the other components. The sheet contribution can be directly evaluated in space domain as follows:

$$
\begin{aligned}
Z_{I B C}^{x x}\left(m, n ; m^{\prime}, n^{\prime}\right)= & \int_{0}^{a} F_{m, n}\left(\frac{\rho}{a}\right) F_{m^{\prime}, n^{\prime}}\left(\frac{\rho}{a}\right) \\
& \int_{0}^{2 \pi} e^{j\left(n-n^{\prime}\right) \phi} Z_{S}^{x x}(\vec{\rho}) d \phi \rho d \rho
\end{aligned}
$$

Thanks to the azimuthal periodicity, it is better to expand $Z_{S}^{x x}$ into a Fourier series as follows:

$$
Z_{S}^{x x}(\vec{\rho})=\sum_{r=-\infty}^{\infty} a_{r}(\rho) e^{j r \phi}
$$

Then (19) reduces to:

$$
\begin{array}{r}
Z_{I B C}^{x x}\left(m, n ; m^{\prime}, n^{\prime}\right)=2 \pi \int_{0}^{a} F_{m, n}\left(\frac{\rho}{a}\right) F_{m^{\prime}, n^{\prime}}\left(\frac{\rho}{a}\right) \\
a_{n^{\prime}-n}(\rho) \rho d \rho
\end{array}
$$

In practice, the first 10 harmonics of the Fourier series are sufficient to describe accurately the impedance. It means that this integral can be assumed equal to zero for $\left|n^{\prime}-n\right|$ large enough.

Those calculations can be organized differently, in a faster way, but requiring more memory. It consists of directly projecting the sheet impedance in the FBBF basis as follows:

$$
Z_{S}^{x x}(\vec{\rho})=\sum_{n^{\prime \prime}=-N}^{N^{\prime \prime}} \sum_{m^{\prime \prime}=1}^{M^{\prime \prime}} \gamma_{n^{\prime \prime} m^{\prime \prime}} R_{m^{\prime \prime}}^{n^{\prime \prime}}\left(\frac{\rho}{a}, \phi\right)
$$

The expansion coefficients are calculated by simple projection:

$$
\begin{array}{r}
\gamma_{n^{\prime \prime} m^{\prime \prime}}=\frac{1}{K\left(m^{\prime \prime}, n^{\prime \prime}\right)} \int_{0}^{2 \pi} \int_{0}^{a} Z_{S}^{x x}(\vec{\rho}) R_{m^{\prime \prime}, n^{\prime \prime}}^{*} \\
\left(\frac{\rho}{a}, \phi\right) \\
\rho d \rho d \phi
\end{array}
$$

where $K$ is a normalization factor given by:

$$
K\left(m^{\prime \prime}, n^{\prime \prime}\right)=-\pi J_{n^{\prime \prime}-1}\left(\lambda_{n^{\prime \prime}}^{m^{\prime \prime}}\right) J_{n^{\prime \prime}+1}\left(\lambda_{n^{\prime \prime}}^{m^{\prime \prime}}\right) a^{2}
$$

After inserting (22) into (19) and integrating along $\phi$, we get:

$$
\begin{gathered}
Z_{I B C}^{x x}\left(m, n ; m^{\prime}, n^{\prime}\right)=2 \pi a^{2} \sum_{m^{\prime \prime}=1}^{M^{\prime \prime}} \gamma_{\left(n^{\prime}-n\right) m^{\prime \prime}} \\
{\left[\int_{0}^{1} F_{m, n}(\rho) F_{m^{\prime}, n^{\prime}}(\rho) F_{m^{\prime \prime},\left(n^{\prime}-n\right)}(\rho) \rho d \rho\right]}
\end{gathered}
$$

The expression between brackets is a unique function of $\left(m, n ; m^{\prime}, n^{\prime} ; m^{\prime \prime}\right)$ and can therefore be tabulated.

\section{Excitation contribution}

The MTS presented in this paper are antennas excited at the origin by an elementary vertical dipole. The Green's function associated to this excitation can be written in the following form:

$$
\tilde{G}_{x z}^{E J}(\vec{k})=\cos (\alpha) \tilde{f}\left(k_{\rho}\right) ; \tilde{G}_{y z}^{E J}(\vec{k})=\sin (\alpha) \tilde{f}\left(k_{\rho}\right)
$$

Inserting this expression into (14), and integrating along $\alpha$ leads to the following expressions:

$$
\begin{gathered}
v_{x}(m, n)=\frac{(-j)^{n}}{2} a^{2} \lambda_{n}^{m} J_{n-1}\left(\lambda_{n}^{m}\right)\left(\delta_{n, 1}+\delta_{n,-1}\right) \\
\int_{0}^{\infty} \frac{J_{n}\left(k_{\rho} a\right)}{\left(\left(\lambda_{n}^{m}\right)^{2}-\left(k_{\rho} a\right)^{2}\right)} \tilde{f}\left(k_{\rho}\right) k_{\rho} d k_{\rho} \\
v_{y}(m, n)=\frac{(-j)^{n}}{2 j} a^{2} \lambda_{n}^{m} J_{n-1}\left(\lambda_{n}^{m}\right)\left(\delta_{n,-1}-\delta_{n, 1}\right) \\
\int_{0}^{\infty} \frac{J_{n}\left(k_{\rho} a\right)}{\left(\left(\lambda_{n}^{m}\right)^{2}-\left(k_{\rho} a\right)^{2}\right)} \tilde{f}\left(k_{\rho}\right) k_{\rho} d k_{\rho}
\end{gathered}
$$

\section{Polar formulation of the current distribution}

The precedent formulation based on the Cartesian $\left(\hat{a}_{x}, \hat{a}_{y}\right)$ current expansion leads to a system of equations that can be written in matrix form:

$$
\left(\underline{\underline{Z}}_{G}-\underline{\underline{Z}}_{I B C}\right) \underline{I}=\underline{V}
$$

where $\underline{\underline{Z}}_{G}$ is the substrate impedance contribution, $\underline{\underline{Z}}_{I B C}$ is the sheet impedance tensor written in Cartesian coordinates. The $\left(\hat{a}_{\rho}, \hat{a}_{\phi}\right)$ formulation can be written in a similar form:

$$
\left(\underline{\underline{Z}}_{G}^{\prime}-\underline{\underline{Z}}_{I B C}\right) \underline{I^{\prime}}=\underline{V^{\prime}}
$$

where $\underline{\underline{Z}}_{G}$ is the equivalent substrate contribution and ${\underline{\underline{Z^{\prime}}}}_{I B C}$ is the $\overline{\overline{s h}} G$ eet contribution directly written in polar $\left(\overline{\hat{a}}_{\rho}, \hat{a}_{\phi}\right)$ coordinates, $\underline{I}^{\prime}$ is the current expansion in the $\left(\hat{a}_{\rho}, \hat{a}_{\phi}\right)$ unit vectors and $\underline{V^{\prime}}$ is the excitation.

Starting from equation (8) and expanding the basis and testing functions in the $\left(\hat{a}_{\rho}, \hat{a}_{\phi}\right)$ basis and test functions, it can be proven that:

$$
\underline{\underline{Z}}_{G}^{\prime}=Q^{H} \underline{\underline{Z}}_{G} Q
$$

and

$$
\underline{V^{\prime}}=Q^{H} \underline{V}
$$

where superscript $\left({ }^{H}\right)$ in (31) and (32) represents the Hermitian transposed.

The matrix $\mathrm{Q}$ can be written in the following form:

$$
\left[\begin{array}{ll}
{\left[Q^{\alpha}\right]} & {\left[Q^{\gamma}\right]} \\
{\left[Q^{\beta}\right]} & {\left[Q^{\alpha}\right]}
\end{array}\right]
$$

Each block of the $Q$ matrix has a size $\left(J_{M A X} * J_{M A X}^{\prime}\right)$, where $J_{M A X}=(2 N+1) * M$ is the number of basis / testing functions used in the $\left(\hat{a}_{x}, \hat{a}_{y}\right)$ formulation and $J_{M A X}^{\prime}=$ 
$\left(2 N^{\prime}+1\right) * M^{\prime}$ is the number of basis / testing functions used in the $\left(\hat{a}_{\rho}, \hat{a}_{\phi}\right)$ formulation. The block $Q^{\alpha}$ is defined as:

$$
\begin{array}{r}
Q^{\alpha}\left(m, n ; m^{\prime}, n^{\prime}\right)=\frac{1}{K(m, n)} \int_{0}^{2 \pi} \int_{0}^{a} R_{m, n}^{x, *}\left(\frac{\rho}{a}, \phi\right) \cos (\phi) \\
R_{m^{\prime}, n^{\prime}}^{x}\left(\frac{\rho}{a}, \phi\right) \rho d \rho d \phi
\end{array}
$$

where $\mathrm{K}$ is a normalization factor given by:

$$
K(m, n)=-\pi J_{n-1}\left(\lambda_{n}^{m}\right) J_{n+1}\left(\lambda_{n}^{m}\right) a^{2}
$$

The expressions of $Q^{\beta}$ and $Q^{\gamma}$, are obtained by replacing the $\cos (\phi)$ in expression (34) by respectively $\sin (\phi)$ and $-\sin (\phi)$. After integrating (34) along $\phi$, we obtain:

$$
\begin{aligned}
Q^{\alpha}\left(m, n ; m^{\prime}, n^{\prime}\right)= & \frac{\pi a^{2}}{K(m, n)}\left(\delta_{n, n^{\prime}-1}+\delta_{n, n^{\prime}+1}\right) \\
& \int_{0}^{1} F_{m, n}(\rho) F_{m^{\prime}, n^{\prime}}(\rho) \rho d \rho
\end{aligned}
$$

Similar expressions are obtained for the others blocks (See Appendix B). The obtained matrix projection $Q$ is then very sparse and can be computed extremely fast. In practice, choosing $J_{M A X}=J_{M A X}^{\prime}$ is sufficient to describe accurately the current projection. In passing, if one is interested only by the $\hat{a}_{\rho}$ oriented currents (as is the case for isotropic MTS antennas), the projection matrix $Q$ is given by:

$$
\left[\begin{array}{l}
{\left[Q^{\alpha}\right]} \\
\left.\left[Q^{\beta}\right]\right]
\end{array}\right]
$$

\section{NUMERICAL RESULTS}

To test the accuracy and the efficiency of the method, we analyze three MTS antennas. The first one is synthesized using an isotropic MTS, i.e. a scalar IBC distribution, whereas the other ones use anisotropic MTSs, i.e. a tensorial IBC distribution. We analyze the FBBFs in terms of convergence (number of basis functions needed to get a stable solution) and computation time, and compare the obtained results with the simulation of the IBC using GRBFs [25], and with the full-wave analysis of MTS antennas implemented with small printed elements.

\section{A. Broadside pencil beam antenna with isotropic MTS}

The analyzed structure is a scalar modulated MTS antenna, designed to radiate a broadside right-handed circularly polarized (RHCP) pencil beam. The design procedure has been discussed in [11] and leads to the following opaque IBC distribution:

$$
\begin{aligned}
& \mathrm{Z}_{+}^{\rho \rho}(\vec{\rho})=j X_{0}\left[1+M_{0} \sin (2 \pi \rho / d-\phi)\right] \\
& \mathrm{Z}_{+}^{\rho \phi}(\vec{\rho})=\mathrm{Z}_{+}^{\phi \rho}(\vec{\rho})=0 \\
& \mathrm{Z}_{+}^{\phi \phi}(\vec{\rho})=j X_{0}\left[1+M_{0} \sin (2 \pi \rho / d-\phi)\right]
\end{aligned}
$$

where: $X_{0}=0.71 \eta_{0}$, with $\eta_{0}$ being the free-space impedance, $M_{0}=0.27$ and $d=\lambda / \sqrt{1+\left(X_{0} / \eta_{0}\right)^{2}}$. The frequency of operation is $f=17 \mathrm{GHz}$ and the antenna radius is $a=5.65 \lambda$ at that frequency. Finally, the dielectric substrate has relative permittivity $\epsilon_{r}=3.66$ and thickness $h=1.524 \mathrm{~mm}$. The sheet impedance is obtained after removing from the opaque one the substrate contribution, as explained in [31], and the MTS is excited with a vertical electric dipole placed in the middle of the substrate and at the center of the disk $(\rho=0)$.

Fig. 4(a) and Fig. 4(b) show the absolute value of the current distribution obtained with FBBF and that obtained with GRBF, respectively. In both simulations, we have used the same number of basis functions $(N=8 ; M=46)$, which leads to a total of 1632 basis functions. We can observe the

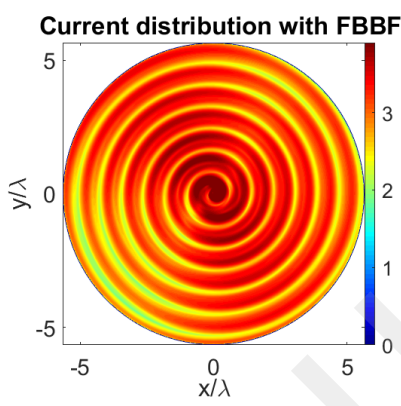

(a)

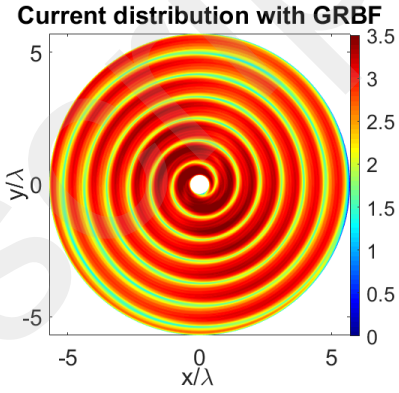

(b)
Fig. 4: Absolute value of the surface current distribution (in $\log 10$ scale) on a scalar IBC aperture used to generate a RHCP broadside pencil-beam. The IBC disk has been analyzed with a) FBBFs and b) GRBFs, in both cases $N=8$ and $M=46$

same appearance in both cases, except close to the center (for $\rho<0.5 \lambda$ ) given that we have not defined GRBFs in the central region. In this way, one may account for the absence of MTS elements often encountered in the region occupied by the feeder. Indeed, in practice, the feed is not a simple vertical dipole and realistic feeds cover a disk of radius about $0.5 \lambda$. On that disk, in the implementation of [11], there are no equivalent electric currents since there are no patches implementing the IBC.

Fig. 5(a) shows the normalized co-polar pattern obtained with GRBF and FBBF. One can observe a difference, especially on the secondary lobes. Indeed, close to the center, the current increases very rapidly, leading to a non-negligible contribution to the far field. This may be viewed as a weakness of the FBBF representation which produce nonzero fields everywhere including close to the feed point. This problem can be partly corrected by removing a posteriori the center currents contribution (in the region where the GRBFs are not defined) from the pattern. The obtained pattern is now represented in Fig. 5(b). A better agreement is observed although it is not perfect since truncating is not strictly equivalent to the use of basis functions which do not admit center currents. The same observation is valid for the cross polar patterns (see Fig. 6). In terms of antenna performances, the results are summarized in Table I. As expected, the performances are much closer to the GRBFs simulation after the center current subtraction. The difference w.r.t the GRBFs Co-polar pattern is $1.5 \mathrm{~dB}$ without center current subtraction and $0.6 \mathrm{~dB}$ after center current subtraction. The remaining error may be attributed to the fact the two simulated structures are not exactly the same, one of them having a zero sheet admittance over a disk of radius $0.5 \lambda$ at the center. Nevertheless, the results obtained 


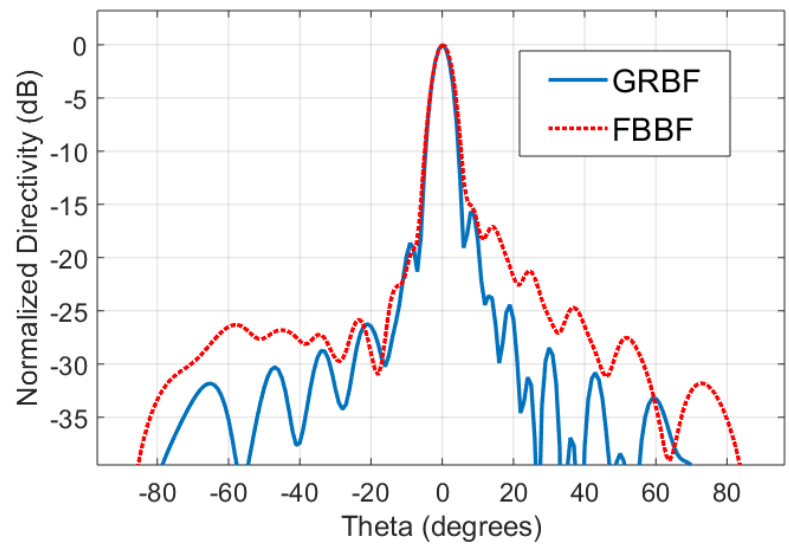

(a)

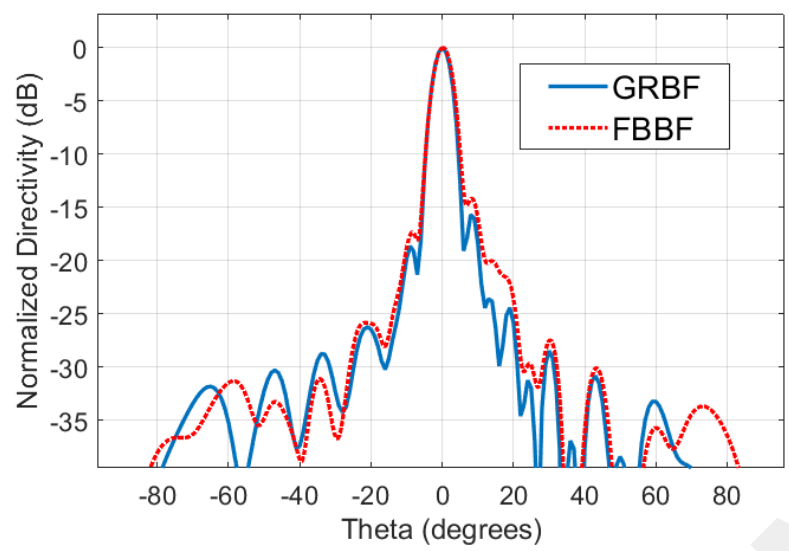

(b)

Fig. 5: RHCP component of the normalized directivity pattern for the structure described by (38). The result obtained with GRBFs (solid line) is compared with the FBBFs one (dashed line) in two cases: a) without center current subtraction and b) with center current subtraction.

TABLE I: Computed Co-polar and Cross-polar maximum directivity for the MTS described by (38).

\begin{tabular}{crc}
\hline Solver & RHCP & LHCP \\
\hline GRBF & $26.5 \mathrm{dBi}$ & $18.2 \mathrm{dBi}$ \\
FBBF without center current subtraction & $25 \mathrm{dBi}$ & $16.6 \mathrm{dBi}$ \\
FBBF with center current subtraction & $25.9 \mathrm{dBi}$ & $18 \mathrm{dBi}$ \\
Difference w.r.t GRBF & $0.6 \mathrm{~dB}$ & $0.2 \mathrm{~dB}$ \\
\hline
\end{tabular}

after center current subtraction provide a good estimation of the radiation performance, both for the Co-polar and the Crosspolar patterns. Next, we have implemented with square patches a smaller version of the MTS ( $3 \lambda$ radius). The patches cover all the disk and the structure is excited with the same vertical dipole. The implemented MTS made of square patches is meshed with 154868 RWG basis functions and simulated with the MoM technique in [41]. Fig. 7 shows the obtained co-polar pattern.

One can observe a good agreement, despite the fact that we did not remove the center current contribution since the implemented MTS is defined on all the disk. It is important to note that for MTSs excited outside the surface or near its

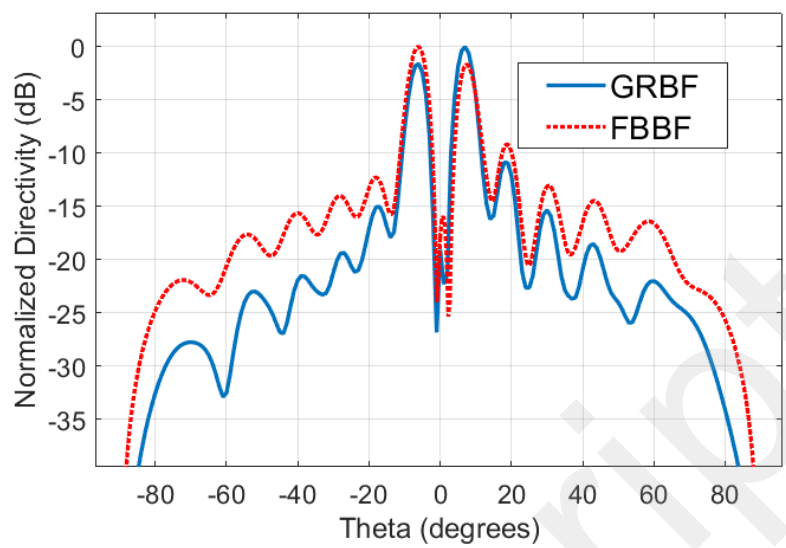

(a)

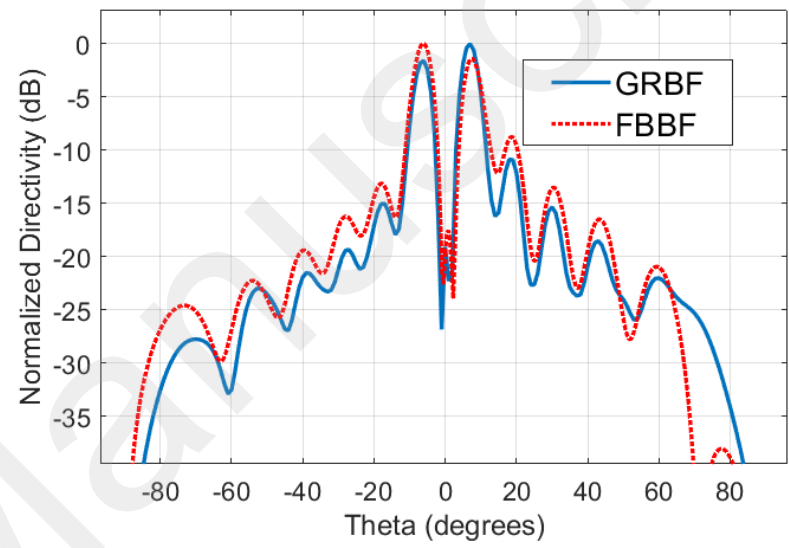

(b)

Fig. 6: LHCP component of the normalized directivity pattern for the structure described by (38). The result obtained with GRBFs (solid line) is compared with the FBBFs one (dashed line) in two cases: a) without center current subtraction and b) with center current subtraction.

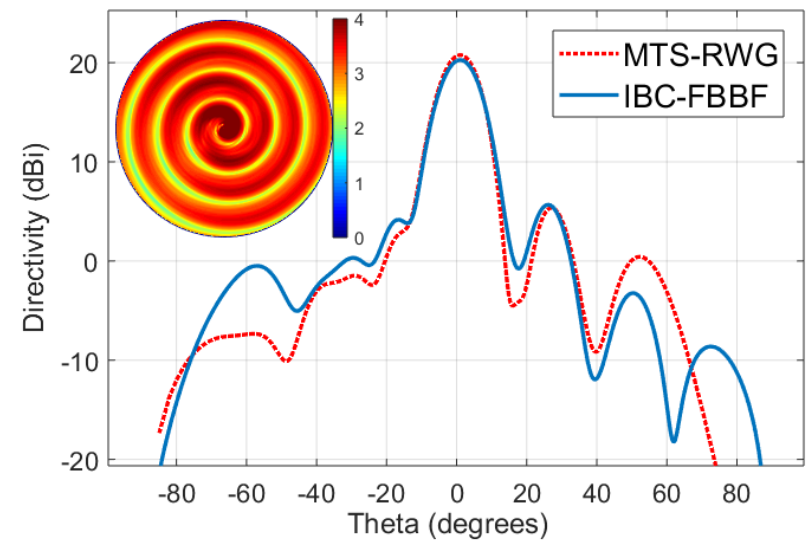

Fig. 7: Directivity pattern without direct excitation contribution of a smaller version of the structure described by (38) and used to obtain a broadside beam. The RHCP pattern obtained with the RWG-MoM simulation of the MTS antenna implemented with square patches (red dotted line) is compared with the pattern obtained using FBBFs and the IBC in the circular domain (blue solid line). The inset disk corresponds to the absolute value of the current distribution (in $\log 10$ scale) obtained with FBBF.

border, as is the case for the Luneburg Lens in [40], the IBC is defined on all the circular domain, thus the FBBFs directly provide good results. However, a full modeling of the MTS 
in presence of realistic feed will require a full-wave analysis of the feeding structure together with its immediate vicinity, taking into account the local coupling between the feed and the IBC.

Below, we study the convergence of the solution (38) obtained with FBBFs, and compare it with that obtained with GRBFs. Both types of function present identical azimuthal dependence. Therefore, we will just compare their convergence rate with respect to the index $M$, which controls the radial discretization. To this end, we analyze the evolution of the pattern shape. We define the relative error for a given number $M$ of radial basis functions as follows:

$$
\operatorname{err}_{M}=100 \frac{\sum_{\theta=0^{\circ}}^{90^{\circ}} \sum_{\phi=0^{\circ}}^{360^{\circ}}\left\|\vec{E}_{M}(\theta, \phi)-\vec{E}_{M+2}(\theta, \phi)\right\|_{2}}{\sum_{\theta=0^{\circ}}^{90^{\circ}} \sum_{\phi=0^{\circ}}^{360^{\circ}}\left\|\vec{E}_{M+2}(\theta, \phi)\right\|_{2}}
$$

The evolution of the relative error obtained with both FBBFs and GRBFs is depicted in Fig. 8. Although the convergence

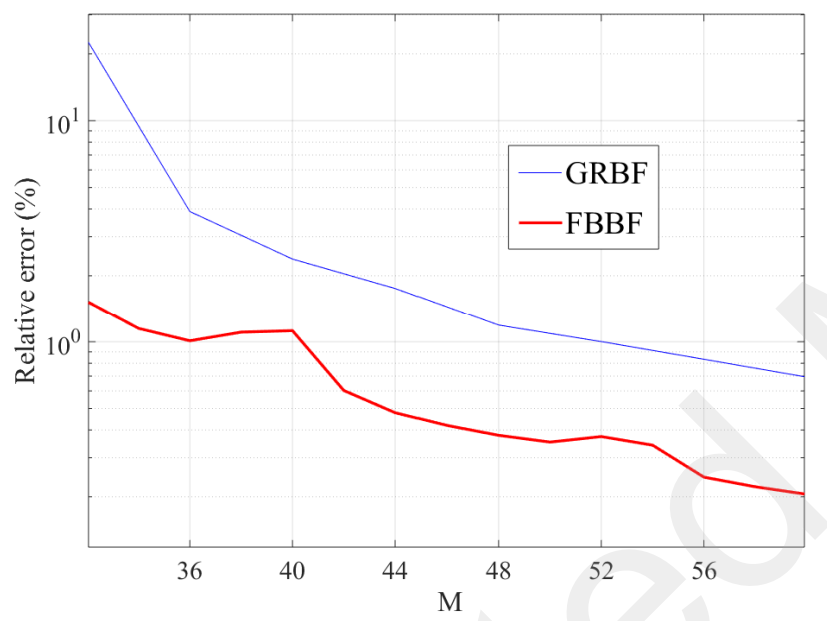

Fig. 8: Relative error versus index $M$ obtained with (39) for the FBBF and the GRBF simulation of the structure in Fig. 4 and Fig. 5.

rate is approximately the same, the number of basis functions needed to obtain a given relative error is smaller when using FBBFs. In particular, for a relative error of approximately $1 \%$, we need for FBBFs, $M=36$ and for GRBF, $M=48$ where $M$ has been defined in (5). As it will be seen later, this difference is more significant for larger MTS. Moreover, for the isotropic MTS case, the orthogonality of the basis allows one to apply the MoM formulation which uses only $\hat{a}_{\rho}$ directed currents, leading to significantly lower number of basis functions. In this particular example, we only need $612 \mathrm{FBBF}$ and $1632 \mathrm{GRBFs}$, provided that we use only $\hat{a}_{\rho}$ directed currents. In the following sections, we will assume that a stable convergence rate is obtained when the relative error $\approx 1 \%$. The computation time needed for the different steps of the simulation is shown in Table II. In order to be consistent in the comparisons, we use now the $\left(\hat{a}_{x}, \hat{a}_{y}\right)$ formulation for the current distribution. The simulations have been done with an Intel Core i7 @ $2.8 \mathrm{GHz}$ computer. One can observe that the simulation based on GRBFs is faster than that using FBBFs. This difference is mainly due to the
TABLE II: Simulation times for the MTS described by (38).

\begin{tabular}{ccccc} 
Solver & Substrate matrix & IBC matrix & Inversion time & Total time \\
\hline FBBF & $6.54 \mathrm{~s}$ & $1.51 \mathrm{~s}$ & $0.14 \mathrm{~s}$ & $9.34 \mathrm{~s}$ \\
& & & & \\
GRBF & $2.59 \mathrm{~s}$ & $0.32 \mathrm{~s}$ & $0.14 \mathrm{~s}$ & $3.58 \mathrm{~s}$ \\
\hline
\end{tabular}

closed-form evaluation of the integrals arising in the GRBF formulation [25]. From the times in Table I, one can conclude that the largest difference lies in the calculation of the substrate matrix $\underline{\underline{Z}}_{G}$, which cannot be computed in closed form with FBBFs. However, in an optimization process, the substrate matrix needs to be calculated only once and for all, while the matrix related to the sheet transition IBC $\underline{\underline{Z}}_{I B C}$ has to be computed at each optimization step. We can also observe that the computation times of the sheet matrix, remains lower for GRBF than for FBBF. This is due to the fact that the evolution of the sheet is smooth in comparison with the Gaussian width, allowing an asymptotic calculation of the reaction integrals. Finally, it is important to mention that the same simulation takes $2.4 \mathrm{~h}$ with FEKO [42], using RWG basis functions and needs 54907 basis functions.

\section{B. Broadside pencil beam MTS antennas based on anisotropic IBCs}

In this subsection, we analyze two anisotropic MTS antennas of different sizes with a RHCP broadside pencil beam. The elements of the impenetrable IBC tensor of the first one are defined as:

$$
\begin{aligned}
\mathrm{Z}_{+}^{\rho \rho}(\vec{\rho}) & =j X_{0}\left[1+M_{\rho \rho} \cos (2 \pi \rho / d-\phi)\right] \\
\mathrm{Z}_{+}^{\rho \phi}(\vec{\rho}) & =\mathrm{Z}_{+}^{\phi \rho}(\vec{\rho})=j X_{0} M_{\rho \phi} \sin (2 \pi \rho / d-\phi) \\
\mathrm{Z}_{+}^{\phi \phi}(\vec{\rho}) & =j X_{0}\left[1-M_{\phi \phi} \cos (2 \pi \rho / d-\phi)\right]
\end{aligned}
$$

where the modulation parameters are $X_{0}=279 \Omega, M_{\rho \rho}=$ $M_{\rho \phi}=M_{\phi \phi}=0.4$ and $d=\lambda / \sqrt{1+\left(X_{0} / \eta_{0}\right)^{2}}$. The frequency of operation is $f=8.425 \mathrm{GHz}$ and the antenna radius is $a=7.6 \lambda$ at this frequency. The cladding lies on a dielectric substrate which has a relative permittivity $\epsilon_{r}=9.8$ and thickness $h=1.57 \mathrm{~mm}$. The obtained current distributions using FBBFs and GRBFs are depicted in Fig. 9(a) and Fig. 9(b), respectively. In both simulations we have used the same discretization parameters with $N=16$ and $M=96$, which leads to a total of 6336 unknowns. Fig. 9(c) presents the evolution of the currents obtained with both methods on the $\phi=0$ axis. We can observe a very good agreement up to $\rho=0.5 \lambda$ since the GRBFs have not been defined in the center region. Fig. 10 shows the current distribution with FBBFs obtained with $60 \%$ fewer basis functions $(N=16 ; M=60$, i.e. 3960 basis functions) in comparison with $(N=16$; $M=96$, i.e. 6336 basis functions). We can still observe a very good correspondence despite, using 60\% fewer FBBFs (3960 vs. 6336). For this example, choosing ( $N=16$; $M=65$ i.e. 4290 basis functions) is enough to get a stable convergence. The calculation times, are summarized in Table II. The same simulation, using RWG basis functions to mesh the patches 


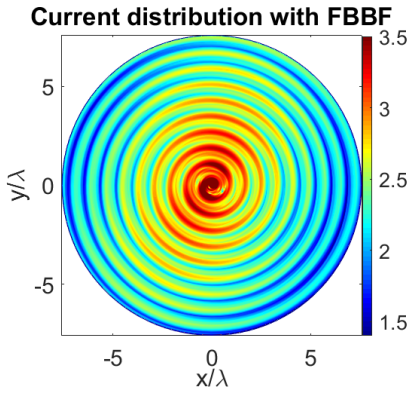

(a)

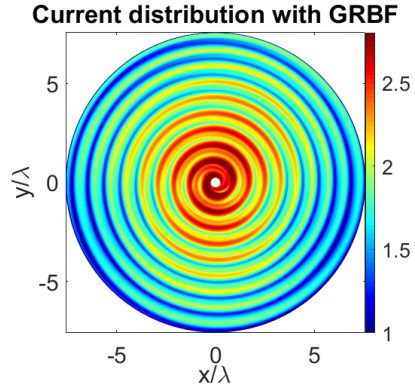

(b)

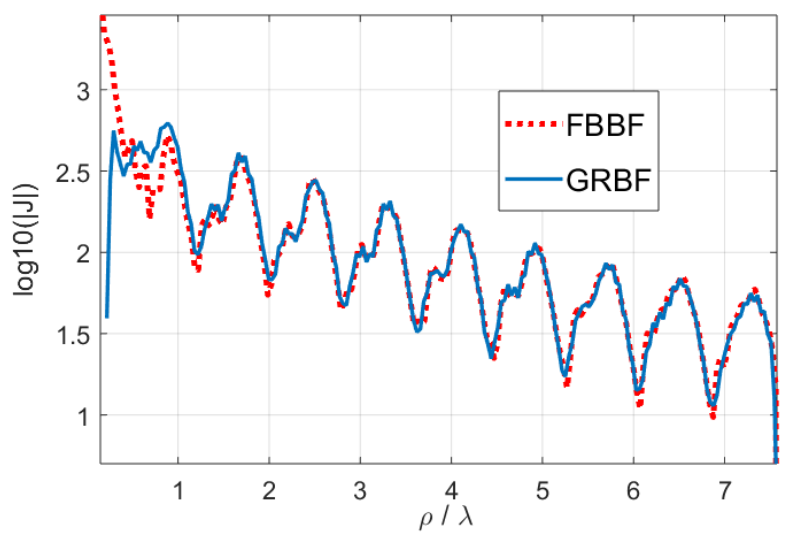

(c)

Fig. 9: Results for the RHCP broadside pencil-beam antenna described by the anisotropic IBC in (40). The IBC disk has been analyzed using $N=16$ and $M=96$. Absolute value of the surface current distribution obtained: a) on the aperture with FBBFs, b) on the aperture with GRBFs, and c) on the $\phi=0$ axis both with FBBFs and GRBFs.

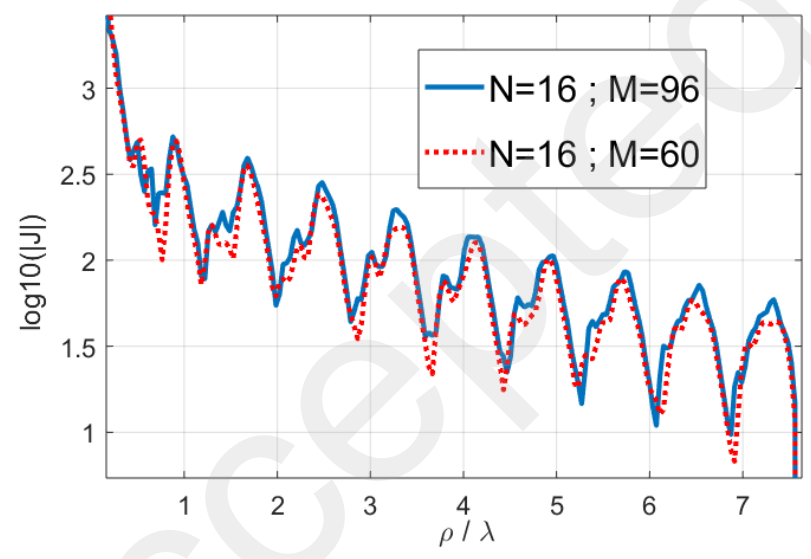

Fig. 10: Convergence analysis for the RHCP broadside pencil-beam antenna described by the anisotropic IBC in (40). Comparison between the absolute value of the currents obtained on the $\phi=0$ axis with FBBFs and $(N=16$; $M=60)$; and with FBBFs and $(N=16 ; M=96)$.

implementing the corresponding IBC requires 1083240 basis functions and $1 \mathrm{~h} 35 \mathrm{~min}$ of computation time even with the fast full-wave solver adopted in [11].

Next, we analyze a larger MTS aperture. The elements of the opaque IBC tensor are now given by (40), with $X_{0}=$ $0.55 \eta_{0}, M_{\rho \rho}=0.2, M_{\rho \phi}=0.2, M_{\phi \phi}=0.15$, and $d=$ $\lambda / \sqrt{1+\left(X_{0} / \eta_{0}\right)^{2}}$. The frequency of operation is $f=30 \mathrm{GHz}$ and the antenna radius is $a=30 \lambda$ at this frequency. The
TABLE III: Simulation times for the MTS described by (40).

\begin{tabular}{crrrr} 
Solver & Substrate matrix & IBC matrix & Inversion time & Total time \\
& & & & \\
\hline FBBF & $21.76 \mathrm{~s}$ & $12.32 \mathrm{~s}$ & $3.67 \mathrm{~s}$ & $39.65 \mathrm{~s}$ \\
GRBF & $7.48 \mathrm{~s}$ & $4.44 \mathrm{~s}$ & $3.67 \mathrm{~s}$ & $16.59 \mathrm{~s}$ \\
\hline
\end{tabular}

cladding lies on a dielectric substrate with relative permittivity $\epsilon_{r}=9.8$ and thickness $h=0.425 \mathrm{~mm}$. The obtained patterns, without and with center current subtraction are represented in Fig. 11(a) and Fig. 11(b). One can observe a good agreement

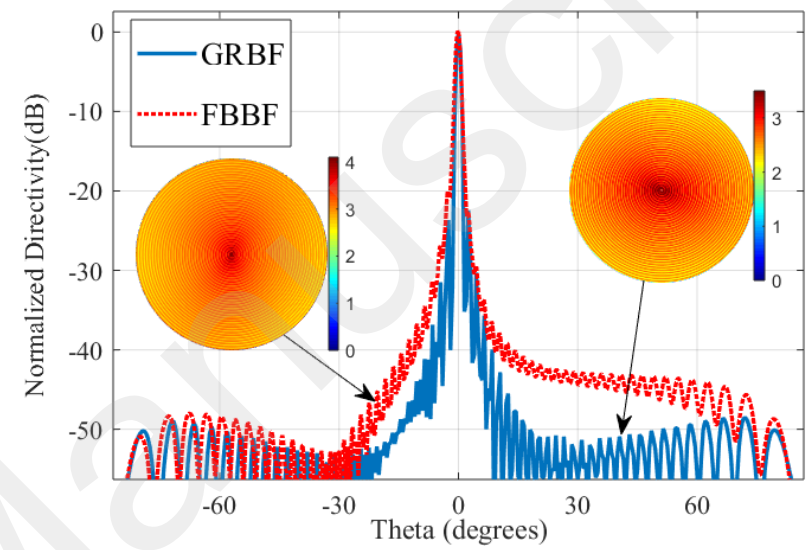

(a)

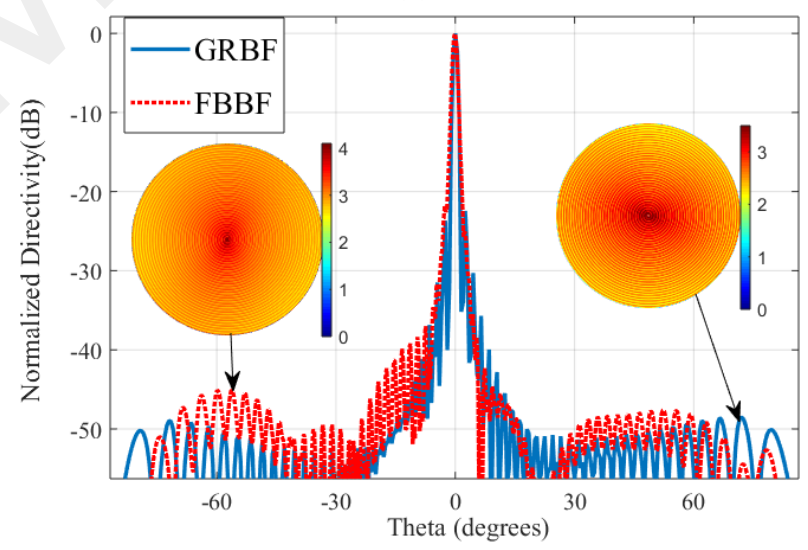

(b)

Fig. 11: RHCP component of the normalized directivity pattern for the structure described by (40). The results obtained with GRBFs (solid line) are compared with the FBBFs one (dashed line) in two cases: a) without center current subtraction and b) with center current subtraction. The inset disk corresponds to the absolute value of the current distribution (in $\log 10$ scale).

between both approaches. However, a stable convergence is obtained with FBBF for $(N=16 ; M=90)$, i.e. 5940 basis functions, whereas in the GRBFs case one needs to use $(N=$ $16 ; M=150$ ) which leads to 9900 basis functions.

\section{Squinted pencil beam MTS antenna based on an anisotropic IBC}

The last example is an anisotropic MTS antenna radiating a RHCP squinted pencil beam at $\theta_{p}=30^{\circ}$ and $\phi_{p}=0^{\circ}$. The 
applied anisotropic IBC is defined as (see Sec. IV-B of [11]):

$$
\begin{aligned}
\mathrm{Z}_{+}^{\rho \rho}(\vec{\rho}) & =j X_{0}\left[1+M_{0} \cos \theta_{p} \cos (2 \pi \rho / d-\gamma-\phi)\right] \\
\mathrm{Z}_{+}^{\rho \phi}(\vec{\rho}) & =\mathrm{Z}_{+}^{\phi \rho}(\vec{\rho})=j X_{0} M_{0} \sin (2 \pi \rho / d-\gamma-\phi) \\
\mathrm{Z}_{+}^{\phi \phi}(\vec{\rho}) & =j X_{0}\left[1-M_{0} \cos (2 \pi \rho / d-\gamma-\phi)\right] / \cos ^{2} \theta_{p}
\end{aligned}
$$

where: $\gamma=k_{0} \rho \cos \phi \sin \theta_{p}$. The other parameters are the same as those considered in section V-B2 of [25]. Here, a stable convergence (relative error $\approx 1 \%$ on the pattern) is obtained with FBBF for $(N=26 ; M=50)$, i.e. 5300 basis functions and with GRBF for $(N=26 ; M=96)$, i.e. 10176 basis functions. The current distributions obtained with FBBFs and GRBFs are represented in Figs. 12(a) and 12(b), respectively. The corresponding co-polar and cross-polar patterns are

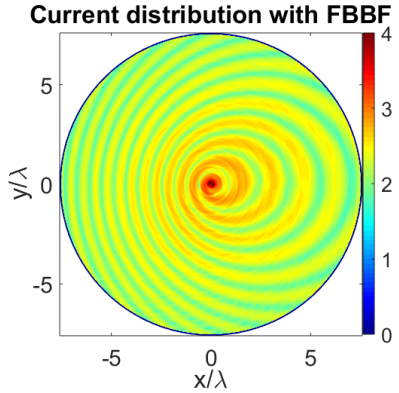

(a)

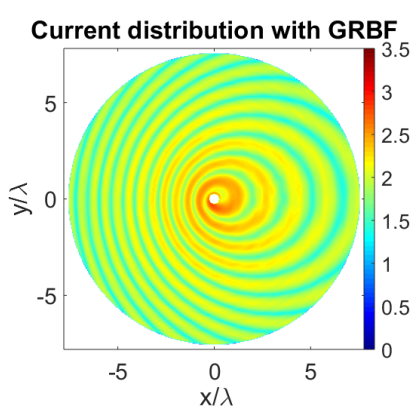

(b)
Fig. 12: Absolute value of the surface current distribution (in $\log 10$ scale) on the anisotropic IBC aperture described by (41) and used to generate a RHCP squinted pencil-beam. The IBC disk has been analyzed with a) FBBFs using $(N=26 ; M=50)$ and with b) GRBFs using $(N=26 ; M=96)$.

depicted in Figs. 13(a) and 13(b), respectively. In the same plots, one can find the radiation pattern from the RWG-MoM simulation of the MTS implemented with screw-head patches in [25]. It is important to note that for the RWG simulation, there are no patches close to the center of the MTS. A good agreement is observed, despite using almost two times fewer basis functions (5300 vs 10176), except for the region close to the MTS center, due to reasons we have already discussed in section IV-A. However, one can see that the radiation pattern obtained after center current subtraction provides a good estimate of the pattern obtained with the RWG-MoM solution of the MTS simulated without center patches. This has been observed for all the simulated examples. Such a subtraction has also proven to produce good comparison with measured data of a MTS antenna made with realistic feed as shown in [17]. The authors believe that such modeling is sufficient to achieve a first MTS antenna design. A fine control of the antenna input impedance and far-out sidelobes may require a full-wave analysis of the feeding structure, taking into account the coupling with the immediate IBC vicinity.

\section{CONCLUSION}

A Method of Moments analysis using Fourier-Bessel Basis Functions (FBBFs) has been proposed to obtain the global evolution of the current distribution on circular modulated metasurfaces. These basis functions are directly defined over the whole computational domain, without any emphasis on

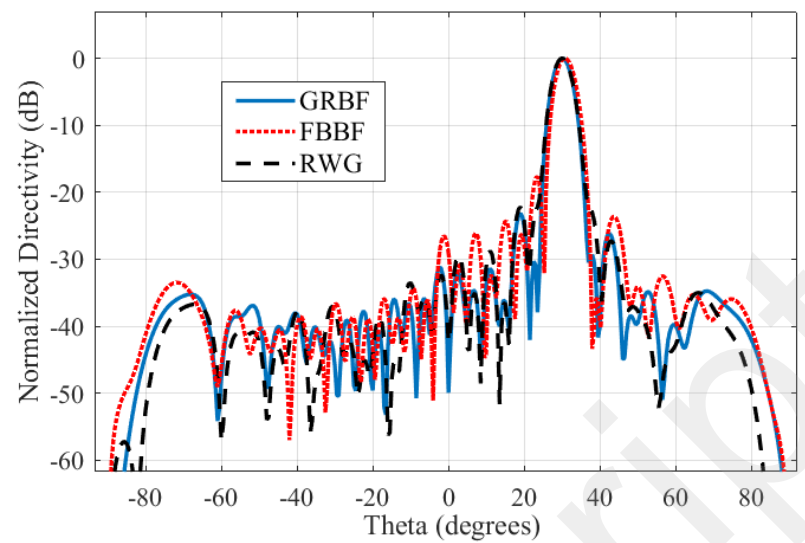

(a)

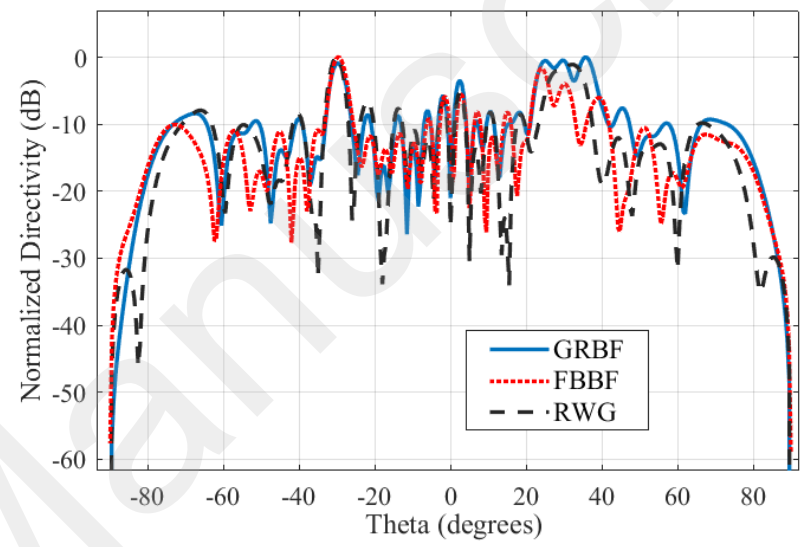

(b)

Fig. 13: Normalized directivity pattern for the structure described by (41) and used to obtain a squinted pencil beam. The results obtained with $N=26$, $M=96$ GRBFs (blue solid line) and those obtained after meshing the patches of the MTS with RWG basis functions are compared with the results obtained using $N=26, M=50$ FBBFs (red dotted line) both for the a) RHCP component and the b) LHCP component.

specific sub-domains. The main advantage of the FourierBessel basis with respect to Gaussian Ring basis Functions (GRBFs) resides in its orthogonality and its completeness, allowing an analysis of a general class of disk current distribution in an effective manner i.e., more compactly. In comparison with the well known family of orthogonal Zernike functions, FBBFs possess a very limited bandwidth, leading to an easy and fast calculation of the reaction integrals in the spectral domain. Moreover we have observed that the Fourier-Bessel basis is better suited than the Zernike basis in describing typical current distributions on circular metasurfaces. The proposed basis can be extended to analyze the more general class of elliptical apertures, while maintaining the orthogonality properties. The basis chosen here is not limited to a particular kind of excitation even if the examples proposed in this paper used a dipole excitation. When the MTS is excited from the center, GRBFs allow a better representation of the currents in the immediate vicinity of the feed. Finally, it is expected that, owing to the orthogonality of the basis, the FBBF decomposition will provide new insight into the design procedure of circular metasurfaces without directly referring to their implementation using patches. 


\section{APPENDIX A}

\section{FOURIER-BESSEL ORTHOGONALITY}

The Bessel functions satisfy the orthogonality relation (for the same $n$ index) on the unit disk:

$$
\begin{aligned}
\int_{0}^{1} F_{m n}\left(\rho_{0}\right) F_{m^{\prime} n}\left(\rho_{0}\right) & \rho_{0} d \rho_{0}=-\delta_{m m^{\prime}} \\
& \frac{J_{n-1}\left(\lambda_{n}^{m}\right) J_{n+1}\left(\lambda_{n}^{m}\right)}{2}
\end{aligned}
$$

After adding the azimuthal term, the Fourier-Bessel functions become fully orthogonal.

$$
\begin{array}{r}
\int_{0}^{2 \pi} \int_{0}^{1} R_{m n}\left(\rho_{0}, \phi\right) R_{m^{\prime} n^{\prime}}^{*}\left(\rho_{0}, \phi\right) \rho_{0} d \rho_{0} d \phi=-\pi \delta_{m m^{\prime}} \delta_{n n^{\prime}} \\
J_{n-1}\left(\lambda_{n}^{m}\right) J_{n+1}\left(\lambda_{n}^{m}\right)
\end{array}
$$

\section{APPENDIX B}

\section{SECOND BLOCK OF THE PROJECTION $Q$ MATRIX}

The $Q^{\beta}$ matrix is defined as follows:

$$
\begin{aligned}
Q^{\beta}\left(m, n ; m^{\prime}, n^{\prime}\right)= & \frac{j \pi a^{2}}{K(m, n)}\left(\delta_{n, n^{\prime}+1}-\delta_{n, n^{\prime}-1}\right) \\
& \int_{0}^{1} F_{m, n}(\rho) F_{m^{\prime}, n^{\prime}}(\rho) \rho d \rho
\end{aligned}
$$

where $K(m, n)$ is given by expression (35) and

$$
Q^{\gamma}\left(m, n ; m^{\prime}, n^{\prime}\right)=-Q^{\beta}\left(m, n ; m^{\prime}, n^{\prime}\right)
$$

\section{ACKNOWLEDGMENTS}

This research has been supported by a FRIA (Fonds pour la formation à la Recherche dans l'Industrie et dans l'Agriculture) grant from the Belgium FNRS (Fonds National de la Recherche Scientifique) fund. The work of D. GonzálezOvejero was supported by a Marie Curie International Outgoing Fellowship within the 7th European Community Framework Programme.

\section{REFERENCES}

[1] C. L. Holloway, A. Dienstfrey, E. F. Kuester, J. F. Ohara, A.K. Azad and A. J. Taylor "A discussion on the interpretation and characterization of metafilms/metasurfaces: The two-dimensional equivalent of metamaterials," Metamaterials, vol.3, no. 2, pp. 100-112, 2009

[2] C. L. Holloway, E. F. Kuester, J. Gordon, J. O'Hara, J. Booth and D. Smith "An overview of the theory and applications of metasurfaces: The twodimensional equivalents of metamaterials," IEEE Antennas Propag. Mag., vol. 54, no. 2, pp. 10-35, 2012.

[3] S. Maci, G. Minatti, M. Casaletti and M. Bosiljevac, "Metasurfing: Addressing waves on impenetrable metasurfaces," IEEE Antennas Wireless Propag. Lett., vol. 10, pp. 1499-1502, Feb. 2011.

[4] N. Yu et al.,"Flat optics: Controlling wavefronts with optical antenna metasurfaces," IEEE J. Sel. Topics Quantum Electron., vol. 19, no. 3, pp. 4700423, May 2013.

[5] C. Pfeiffer, A. Grbic, "Metamaterial huygens' surfaces: Tailoring wave fronts with reflectionless sheets," Phys. Rev. Lett., vol. 110, pp. 197401, May 2013.

[6] R. Quarfoth, D. Sievenpiper, "Artificial tensor impedance surface waveguides," IEEE Trans. Antennas Propag., vol. 61, no. 7, pp. 3597-3606, Jul. 2013.

[7] M. Mencagli, E. Martini, D. González-Ovejero, and S. Maci, "Metasurfing by transformation electromagnetics," IEEE Antennas Wireless Propag. Lett., vol. 13, pp. 1767-1770, Oct. 2014.
[8] A. M. Patel, A. Grbic, "A printed leaky-wave antenna based on a sinusoidally-modulated reactance surface", IEEE Trans. Antennas Propag., vol. 59, no. 6, pp. 2087-2096, Jun. 2011.

[9] D. González-Ovejero, G. Minatti, G. Chattopadhyay and S. Maci, "Multibeam by metasurface antennas," IEEE Trans. Antennas Propag., vol. 65, no. 6, pp. 2923-2930, June 2017.

[10] G. Minatti, S. Maci, P. De Vita, A. Freni, M. Sabbadini, "A circularlypolarized isoflux antenna based on anisotropic metasurface," IEEE Trans. Antennas Propag., vol. 60, pp. 4998-5009, Nov. 2012.

[11] G. Minatti, et al."Modulated metasurface antennas for space: synthesis, analysis and realizations," IEEE Trans. Antennas Propag., vol. 63, pp. 1288-1300, May. 2014.

[12] M. Casaletti, M. Smierzchalski, M. Ettorre, R. Sauleau and N. Capet, "Polarized beams using scalar metasurfaces," IEEE Trans. Antennas Propag., vol. 64, pp. 3391-3400, May. 2016.

[13] B. H. Fong, J. S. Colbrun, J. J. Ottusch, J. L. Visher and D. F. Sievenpiper "Scalar and tensor holographic artificial impedance surfaces," IEEE Trans. Antennas Propag., vol. 58, no. 10 pp. 3212-3221, Oct. 2010.

[14] G. Minatti, F. Caminita, E. Martini, M. Sabbadini and S. Maci, "Synthesis of modulated-metasurface antennas with amplitude, phase, and polarization control," IEEE Trans. Antennas Propag., vol. 64, no. 9 pp. 3907-3919, Sept. 2016.

[15] S. Mahmoud, Y. M. M. Antar, H. Hammad, and A. Freundorfer, "Theoretical considerations in the optimization of surface waves on a planar structure," IEEE Trans. Antennas Propag., vol. 52, no. 8 pp. 20572063, Aug. 2004.

[16] H. Bui-Van, C. Craeye, E. de Lera Acedo, "Main beam modeling for large irregular Arrays: The SKA1-LOW telescope case," Exp. Astronom., vol. 44, pp. 239-258, 2017.

[17] M. Bodehou, C. Craeye, H. Bui-Van, and I. Huynen, "Fourier-Bessel basis functions for the analysis of elliptical domain metasurface antennas," IEEE Antennas Wireless Propag. Lett., vol. 17, pp. 675-678, April. 2018.

[18] M. Bercigli, et al. , "Hybrid SFX/MLayAIM method for the analysis and optimization of large reflectarrays and planar arrays with metallic lenses," 4th Eur. Conf. Antennas Propag. (EuCAP), 12-16 April 2010.

[19] M. Mencagli, E. Martini, and S. Maci, "Surface wave dispersion for anisotropic metasurfaces constituted by elliptical patches," IEEE Trans. Antennas Propag., vol. 63, no. 7 pp. 2992-3003, Jul. 2015.

[20] D. González-Ovejero, F. Mesa and C. Craeye, "Accelerated macro basis functions analysis of finite printed antenna arrays through 2D and 3D multipole expansions," IEEE Trans. Antennas Propag., vol. 61, pp. 707717, Feb. 2013

[21] L. Matekovits, V. A. Laza, and G. Vecchi, "Analysis of large complex structures with the synthetic-functions approach," IEEE Trans. Antennas Propag., vol. 55, no. 9 pp. 2509-2521, Sept. 2007.

[22] P. De Vita, et al., "Metasurface-by-design: An emerging concept for antennas and beam forming networks," Antennas and Propagation in Wireless Communications (APWC)., 9-13 Sept. 2013.

[23] B. Stupfel, and D. Poget, "Sufficient uniqueness conditions for the solution of the time harmonic maxwell's equations associated with surface impedance boundary conditions," J. Comput. Phys., vol. 230, no. 12 pp. 4571-4587, Jun. 2011.

[24] G. Minatti, F. Caminita, E. Martini, and S. Maci, "Flat optics for leakywaves on modulated metasurfaces: adiabatic floquet-wave analysis," IEEE Trans. Antennas Propag., vol. 64, no. 9 pp. 3896-3906, Sept. 2016.

[25] D. González-Ovejero and S. Maci, "Gaussian ring basis functions for the analysis of modulated metasurface antennas," IEEE Trans. Antennas Propag., vol. 63, pp. 3982-3993, Sept. 2015.

[26] E. Bleszynski, M. Bleszynski, and T. Jaroszewicz "Surface-integral equations for electromagnetic scattering from impenetrable and penetrable sheets," IEEE Antennas Propag. Mag., vol. 35, no. 6, pp. 14-25, Dec. 1993.

[27] W. Glisson, "Electromagnetic scattering by arbitrarily shaped surfaces with impedance boundary condition", Radio Sci., vol. 27, no. 6, pp. 935943, Nov. 1992.

[28] S. Tretyakov, "Analytical Modeling in Applied Electromagnetics," Norwood, MA, USA: Artech House, 2003.

[29] E. F. Kuester,, M. Mohamed, M. Piket-May, and C. Holloway, "Averaged transition conditions for electromagnetic fields at a metafilm," IEEE Trans. Antennas Propag., vol.51, no. 10 pp. 2641-2651, Oct. 2003.

[30] A. M. Patel, and A. Grbic, "Modeling and analysis of printed-circuit tensor impedance surfaces," IEEE Trans. Antennas Propag., vol. 61, no. 1 pp. 211-220, Jan. 2013.

[31] M.A. Francavilla, E. Martini, S. Maci and G. Vecchi, "On the numerical simulation of metasurfaces with impedance boundary condition integral equations," IEEE Trans. Antennas Propag., vol. 63, pp. 2153-2161, May. 2015. 
[32] Z. Zernike, "Beugungstheorie des schneidenverfahrens," Physica., vol. 1, p. $689,1934$.

[33] M. Born, and E. Wolf "Principles of optics," Pergamon Press., 808 p., Oxford, 1975.

[34] A. Aghasi, H. Amindavar, E.L. Miller and J. Rashed-Mohassel,"Flat-top footprint pattern synthesis through the design of arbitrarily planar-shaped apertures," IEEE Trans. Antennas Propag., vol. 58, pp. 2539-2551, Aug. 2010.

[35] M. Bodehou, D. González-Ovejero, C. Craeye and I. Huynen,"Numerical analysis of modulated metasurface antennas using Fourier-Bessel Basis Functions ," IEEE MTT-S Int. Conf. Numerical Electromagnetic Multiphysics Modeling Optimization, pp. 158-160, 2017.

[36] D .M. Pozar, "Input impedance and mutual coupling of rectangular microstrip antennas," IEEE Trans. Antennas Propag., vol. 30, no. 6, pp. 1191-1196, Nov. 1982.

[37] P. Gay-Balmaz, and J. R. Mosig "Three-dimensional planar radiating structures in stratified media," Int. Journ. Microw. and Millimeter-Wave Computer-Aided Eng., vol. 7, no. 5, pp. 330-343, 1997.

[38] L. Vegni, and R. Cicchetti, and P. Capece "Spectral dyadic green's function formulation for planar integrated structures," IEEE Trans. Antennas Propag., vol. 36, no. 8, pp. 1057-1065, Aug. 1998.

[39] Shambhu Nath Jha, and C. Craeye,"Contour-FFT based spectral domain MBF analysis of large printed antenna Arrays," IEEE Trans. Antennas Propag., vol. 62, no. 11, pp. 5752-5764, Nov. 2014.

[40] M. Bosiljevac, M. Casaletti, F. Caminita, Z. Sipus, and S. Maci,"Nonuniform metasurface Luneburg lens antenna design," IEEE Trans. Antennas Propag., vol. 60, no. 9, pp. 4065-4073, Sep. 2012.

[41] S. Hubert, S. N. Jha, and C. Craeye, "Analysis of large non-regular printed scatterers using the contour-FFT," Accepted for publication in IEEE Trans. Antennas Propag., Aug. 2018.

[42] EM Software and Systems-S.A. (Pty) Ltd. (2014). FEKO: Field computations Involving Bodies of Arbitrary Shape, Stellenbosch, South Africa [Online]. Available: http://www.feko.info.

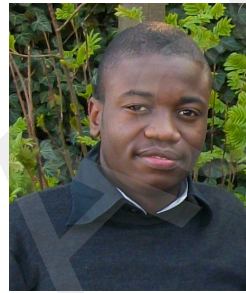

Modeste Bodehou (S'16) was born in Cotonou, Benin, in 1992. He received the B.Sc. degree in engineering and the M.Sc. degree in electrical engineering (telecommunication) from the Royal Military Academy, Brussels, Belgium, in 2013 and 2015, respectively. He is currently pursuing the $\mathrm{Ph} . \mathrm{D}$. degree with the Microwave and Applied Electromagnetism Laboratory, Université catholique de Louvain, Louvain-la-Neuve, Belgium.

In 2015, he was with the Quartier Major Housiau (Belgium Defense), Vilvoorde, Belgium, where he was involved in the framework of his application school on military communication systems. His current research interests include computational electromagnetics, antenna arrays, and metasurfaces.

Mr. Bodehou was a recipient of the First Prize Student Paper Competition Award at the Internantional Conference on Numerical Electromagnetic and Multiphysics Modeling and Optimization for RF, Microwave, and Terahertz Applications in 2017, Seville, and the AIA Price for the Best Engineering Master Thesis Award from the Belgian Royal Military Academy.

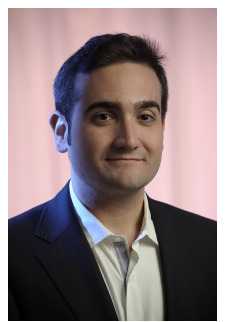

David González-Ovejero (S'01-M'13-SM'17) was born in Gandía, Spain, in 1982. He received the telecommunication engineering degree from the Universidad Politécnica de Valencia, Valencia, Spain, in 2005, and the Ph.D. degree in electrical engineering from the Université catholique de Louvain, Louvainla-Neuve, Belgium, in 2012.

From 2006 to 2007, he was as a Research Assistant with the Universidad Politécnica de Valencia. In 2007, he joined the Universite catholique de Louvain, where he was a Research Assistant until 2012. From 2012 to 2014, he worked as Research Associate at the University of Siena, Siena, Italy. In 2014, he joined the Jet Propulsion Laboratory, California Institute of Technology, Pasadena, CA, USA, where he was a Marie Curie Postdoctoral Fellow until 2016. Since then, he has been a Research Scientist with the French National Center for Scientific Research (CNRS), appointed at the Institut d'Electronique et de Télécommunications de Rennes, France.

Dr. González-Ovejero was a recipient of a Marie Curie International Outgoing Fellowship from the European Commission 2013, the Sergei A. Schelkunoff Transactions Prize Paper Award from the IEEE Antennas and Propagation Society in 2016, and the Best Paper Award in Antenna Design and Applications at the 11th European Conference on Antennas and Propagation in 2017.

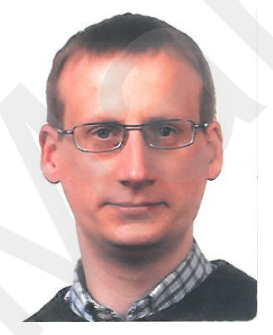

Christophe Craeye (M'98-SM'11) was born in Belgium in 1971. He received the Electrical Engineering and Bachelor in Philosophy degrees and the Ph.D. degree in applied sciences from the Université catholique de Louvain (UCL), Louvain-la-Neuve, Belgium, in 1994 and 1998, respectively. From 1994 to 1999 , he was a Teaching Assistant with UCL and carried out research on the radar signature of the sea surface perturbed by rain, in collaboration with NASA and ESA. From 1999 to 2001, he was a Postdoctoral Researcher with the Eindhoven University of Technology, Eindhoven, The Netherlands. His research there was related to wideband phased arrays devoted to the square kilometer array radio telescope. In this framework, he was also with the University of Massachusetts, Amherst, MA, USA, in the Fall of 1999, and was with the Netherlands Institute for Research in Astronomy, Dwingeloo, The Netherlands, in 2001. In 2002, he started an antenna research activity at the Université catholique de Louvain, where he is now a Professor. He was with the Astrophysics and Detectors Group, University of Cambridge, Cambridge, U.K., from January to August 2011. His research is funded by Région Wallonne, European Commission, ESA, FNRS, and UCL. His research interests include finite antenna arrays, wideband antennas, small antennas, metamaterials, and numerical methods for fields in periodic media, with applications to communication and sensing systems.

Prof. Craeye served as an Associate Editor for the IEEE transactions on antennas and propagation and for the IEEE Antennas and Wireless Propagation Letters from 2011 to 2017. In 2009, he was the recipient of the 2005-2008 Georges Vanderlinden Prize from the Belgian Royal Academy of Sciences.

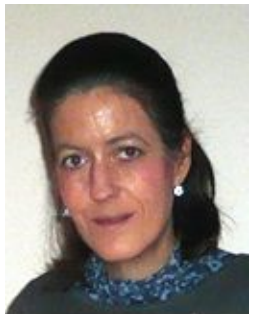

Isabelle Huynen (M'96-SM'06) was born in 1965 in Brussels, Belgium. She earned the $\mathrm{PhD}$ degree in Applied Sciences in 1994 at the Université catholique de Louvain (U.C.L). Since 1999 she is Research Director of the National Fund of Scientific Research and part-time Professor at UCL. Her current research interests cover nanotechnology, nanodevices and nanomaterials for microwave and millimeter wave applications, including metamaterials, antennas and absorbers. 